\title{
On isolated log canonical centers
}

\author{
Chih-Chi Chou
}

\begin{abstract}
In this paper, we show that the depth of an isolated log canonical center is determined by the cohomology of the -1 discrepancy divisors over it. A similar result also holds for normal isolated Du Bois singularities.
\end{abstract}

\section{Introduction}

Singularities play a significant role in the minimal model program (mmp). Among the different types of singularities, Kawamata log terminal (klt) and log canonical (lc) are of particular importance. Many fundamental theorems are first proved in the klt case, then extended to the lc case. And it is expected that lc should be the largest class of singularities for which one can run mmp.

One of the major differences between klt and lc is that klt singularities are rational singularities and lc singularities are Du Bois (see [12]) but in general not rational. So it is interesting and important to know how far lc is from being rational. Since rational implies Cohen-Macaulay, we can also ask if the variety $X$ is Cohen-Macaulay at some given point $p$. Or more precisely, we can calculate $\operatorname{depth}_{p}\left(\mathcal{O}_{X}\right)$.

There are some known results regarding this direction. For example, Fujino shows that given an lc pair $(X, \Delta)$ of dimension at least three, then $\operatorname{depth}_{p}\left(\mathcal{O}_{X}\right) \geq$ $\min \left\{3, \operatorname{codim}_{p} X\right\}$ if $\bar{p}$ is not an lc center (see [6, Theorem 4.21]), which was first proved by Alexeev assuming that $p$ is a closed point and $X$ is projective (see [1, Lemma 3.2]). Kollár and Kovács generalized this result in [10] and [15], respectively, but still under the assumption that $\bar{p}$ is not an lc center (see also [2] for results about closed points).

In this paper, we investigate a case when $\bar{p}$ is an lc center. Assume that $p$ is an isolated lc center; after localization we assume that $p$ is a closed point. It turns out that there is a delicate relation between $\operatorname{depth}_{p}\left(\mathcal{O}_{X}\right)$ and the cohomology group of the exceptional divisors over $p$. More precisely, given an lc pair $(X, \Delta)$ and an isolated lc center $p \in X$ which is a closed point, we take a log resolution $f: Y \rightarrow X$ such that

$$
K_{Y}=f^{*}\left(K_{X}+\Delta\right)+A-B-E
$$

Kyoto Journal of Mathematics, Vol. 54, No. 3 (2014), 497-505

DOI 10.1215/21562261-2693406, (C) 2014 by Kyoto University

Received March 29, 2013. Revised April 9, 2013. Accepted April 12, 2013.

2010 Mathematics Subject Classification: 14B05, $14 \mathrm{~F} 17$. 
Here $A, B$ are effective, $\lfloor B\rfloor=0$, and $E$ is the reduced divisor such that $f(E)=p$. Then we have the following.

THEOREM 1.1 (COROLLARY 3.2)

For any integer $3 \leq t \leq n$, we have $\operatorname{depth}_{p} \mathcal{O}_{X} \geq t$ if and only if $\mathrm{H}^{i-1}\left(E, \mathcal{O}_{E}\right)=0$, $\forall 1<i<t$. (Note that by assumption $X$ is normal, so we know that $\operatorname{depth}_{p} \mathcal{O}_{X}$ is at least two.)

This result generalizes [4, Proposition 4.7], which gives a necessary and sufficient condition for an index one isolated lc singularity to be Cohen-Macaulay.

We prove this theorem by showing that the local cohomology $\mathrm{H}_{p}^{i}\left(\mathcal{O}_{X}\right)$ is the Matlis dual of $\mathrm{H}^{n-i}\left(E, K_{E}\right)$. The same method applies to isolated Du Bois singularities (see Section 3.2). In the Du Bois case, $E$ denotes the reduced exceptional divisors.

The most crucial ingredient of the proof is Kovács's vanishing theorem, which says that $R^{i} f_{*} \mathcal{O}_{Y}(-E)=0, \forall i>0$. With this theorem, we see that $f_{*} \mathcal{O}_{Y}(-E)$ is quasi-isomorphic to $\mathcal{R} f_{*} \mathcal{O}_{Y}(-E)$. By this quasi-isomorphism and Grothendieck duality, we are able to see the relation between the local cohomology of $X$ and the cohomology of $\mathcal{O}_{E}$. Because of the significant role of Kovács's theorem in this paper, we give a quick proof of it in the last section. This proof, based on Fujino's idea, only uses Grothendieck duality and the Kawamata-Viehweg vanishing theorem instead of the notion of Du Bois pairs in Kovács's original paper.

\section{Preliminaries}

Given a pair $(X, \Delta)$, where $X$ is a normal variety and $\Delta$ is a $\mathbb{Q}$-linear combination of Weil divisors so that $K_{X}+\Delta$ is $\mathbb{Q}$-Cartier. Take a log resolution $f: Y \rightarrow X$, such that the exceptional locus and the strict transform $f_{*}^{-1} \Delta$ are simple normal crossing divisors. We say the pair $(X, \Delta)$ is lc if

$$
K_{Y}=f^{*}\left(K_{X}+\Delta\right)+A-B-E,
$$

where $A, B$ are effective, $\lfloor B\rfloor=0$, and $E$ is reduced. We say that $(X, \Delta)$ is $\log$ terminal if $E$ is empty.

In this paper we consider the lc pair $(X, \Delta)$. A subvariety $W \subset X$ is called the lc center, if there is a log resolution as above and some component $E^{\prime} \subset E$ such that $f\left(E^{\prime}\right)=W$.

We recall Kovács's vanishing theorem.

THEOREM 2.1 ([14, THEOREM 1.2])

Let $(X, \Delta)$ be lc pair, and let $f: Y \rightarrow X$ be a proper birational morphism from a smooth variety $Y$ such that $\operatorname{Ex}(f) \cup \operatorname{Supp} f_{*}^{-1} \Delta$ is a simple normal crossing divisor on $Y$. If we write

$$
K_{Y}=f^{*}\left(K_{X}+\Delta\right)+\sum_{i} a_{i} E_{i}
$$


and put $E=\sum_{a_{i}=-1} E_{i}$, then

$$
R^{i} f_{*} \mathcal{O}_{Y}(-E)=0
$$

for every $i>0$.

This theorem is first proved by the notion of Du Bois pairs under the assumption that $X$ is $\mathbb{Q}$-factorial. The proof is then simplified in [5] without assuming $\mathbb{Q}$ factorial.

Now we recall the duality theorems which will be used in this paper. First we recall the Grothendieck duality theorem (see [7, Chapter III Proposition 11.1, Chapter VII Proposition 3.4]). Let $f: Y \rightarrow X$ be a proper morphism between finite-dimensional Noetherian schemes. Suppose that both $X$ and $Y$ admit dualizing complexes, for example, when they are quasiprojective varieties. Then for any $\mathcal{F}^{\bullet} \in D_{\text {qcoh }}^{-}(Y)$, we have

$$
R f_{*} R \mathcal{H} \operatorname{om}_{Y}\left(\mathcal{F}^{\bullet}, \omega_{Y}^{\bullet}\right) \cong R \mathcal{H} o m_{X}\left(R f_{*} \mathcal{F}^{\bullet}, \omega_{X}^{\bullet}\right) .
$$

Here $\omega_{X}^{\bullet}$ is dualizing complex. Let $n$ be the dimension of $X$, and assume that $X$ is normal; then $h^{-n}\left(\omega_{X}^{\bullet}\right):=\omega_{X}=\mathcal{O}_{X}\left(K_{X}\right)$, the extension of regular $n$-forms on smooth locus. In this paper we only consider normal varieties, so we will use $\omega_{X}$ and $K_{X}$ interchangeably. If $X$ is Cohen-Macaulay, then $h^{i}\left(\omega_{X}^{\bullet}\right)=0$, if $i \neq-n$, and $h^{-n}\left(\omega_{X}^{\bullet}\right)=\omega_{X}$. Or equivalently, $\omega_{X}^{\bullet}=\omega_{X}[n]$.

Now we recall local duality (see $[7$, Chapter V, 6.2]). Suppose that $(R, p)$ is a local ring. An injective hull $I$ of the residue field $k=R / p$ is a an injective $R$-module $I$ such that for any nonzero submodule $N \subset I$ we have $N \cap k \neq 0$ (see [3, Proposition 3.2.2] for more discussion). Matlis duality says that the functor $\operatorname{Hom}(\cdot, I)$ is a faithful exact functor on the category of Noetherian $R$-modules.

THEOREM 2.2 (LOCAL DUALITY)

Let $(R, p)$ be a local ring, and let $\mathcal{F}^{\bullet} \in D_{\text {coh }}^{+}(R)$. Then

$$
\mathbf{R} \Gamma_{p}\left(\mathcal{F}^{\bullet}\right) \rightarrow \mathbf{R} \operatorname{Hom}\left(\mathbf{R} \operatorname{Hom}\left(\mathcal{F}^{\bullet}, \omega_{R}^{\bullet}\right), I\right)
$$

is an isomorphism.

In particular, if we take the $i$ th cohomology on both sides, we have

$$
\mathrm{H}_{p}^{i}\left(\mathcal{F}^{\bullet}\right) \cong \operatorname{Hom}\left(\mathrm{H}^{-i}\left(\mathbf{R} \operatorname{Hom}\left(\mathcal{F}^{\bullet}, \omega_{R}^{\bullet}\right)\right), I\right) .
$$

The $-i$ comes from switching the cohomology functor $\mathrm{H}^{i}(\cdot)$ and $\operatorname{Hom}(\cdot, I)$.

\section{Main results}

\subsection{Depth of LC center}

Consider an lc pair $(X, \Delta)$ and an isolated lc center $p \in X$ which is a closed point. Without loss of generality, we assume that $X$ is an affine space and $p$ is the only closed point. By definition, we have a log resolution $f: Y \rightarrow X$ such that

$$
K_{Y}=f^{*}\left(K_{X}+\Delta\right)+A-B-E .
$$


Here $A, B$ are effective, $\lfloor B\rfloor=0, E$ is the reduced exceptional divisor such that $f(E)=p$.

\section{THEOREM 3.1}

For $1<i<n, \mathrm{H}_{p}^{i}\left(X, \mathcal{O}_{X}\right)$ is dual to $\mathrm{H}^{n-i}\left(E, K_{E}\right)$ by Matlis duality. For $i=n$, $\mathrm{H}_{p}^{n}\left(X, \mathcal{O}_{X}\right)$ is dual to $f_{*} \mathcal{O}_{Y}\left(K_{Y}+E\right)$.

Proof

Push forward the following exact sequence on $Y$ :

$$
0 \rightarrow K_{Y} \rightarrow K_{Y}(E) \rightarrow K_{E} \rightarrow 0 .
$$

By Grauert-Riemenschneider vanishing, we have $R^{n-i} f_{*} \mathcal{O}_{Y}\left(K_{Y}+E\right) \cong$ $\mathrm{H}^{n-i}\left(E, K_{E}\right)$ for $i<n$. So to prove the statement, it suffices to prove the duality between $\mathrm{H}_{p}^{i}\left(X, \mathcal{O}_{X}\right)$ and $R^{n-i} f_{*} \mathcal{O}_{Y}\left(K_{Y}+E\right) \cong \mathrm{H}^{n-i}\left(E, K_{E}\right)$. To this end, we consider the quasi-isomorphism $f_{*} \mathcal{O}_{Y}(-E) \cong{ }_{\text {quasi }} \mathbf{R} f_{*} \mathcal{O}_{Y}(-E)$ implied by Kovács's vanishing theorem. Applying Grothendieck duality, we have

$$
\mathbf{R} \operatorname{Hom}\left(f_{*} \mathcal{O}_{Y}(-E), \omega_{X}^{\bullet}\right) \cong_{\text {quasi }} \mathbf{R} \operatorname{Hom}\left(\mathbf{R} f_{*} \mathcal{O}_{Y}(-E), \omega_{X}^{\bullet}\right) \cong_{\text {quasi }} \mathbf{R} f_{*} \omega_{Y}^{\bullet}(E) \text {. }
$$

Taking the $-i$ th cohomology, we have

$$
\operatorname{Ext}^{-i}\left(f_{*} \mathcal{O}_{Y}(-E), \omega_{X}^{\bullet}\right) \cong R^{n-i} f_{*} \mathcal{O}_{Y}\left(K_{Y}+E\right) .
$$

By Matlis duality, the left-hand side is isomorphic to $\operatorname{Hom}\left(\mathrm{H}_{p}^{i}\left(f_{*} \mathcal{O}_{Y}(-E)\right), I\right)$, where $I$ is the injective hull of $k$.

To prove the statement, we claim that $\mathrm{H}_{p}^{i}\left(f_{*} \mathcal{O}_{Y}(-E)\right) \cong \mathrm{H}_{p}^{i}\left(\mathcal{O}_{X}\right)$ for $i>1$. This follows from the exact sequence

$$
0 \rightarrow f_{*} \mathcal{O}_{Y}(-E) \rightarrow \mathcal{O}_{X} \rightarrow \mathcal{O}_{p} \rightarrow 0
$$

and the fact that $\mathrm{H}^{i}\left(\mathcal{O}_{p}\right)=0$ if and only if $i>0$.

\section{COROLLARY 3.2}

For any integer $3 \leq t \leq n$, we have $\operatorname{depth}_{p} \mathcal{O}_{X} \geq t$ if and only if $\mathrm{H}^{i-1}\left(E, \mathcal{O}_{E}\right)=0$, $\forall 1<i<t$. (Note that by assumption $X$ is normal, so we know that $\operatorname{depth}_{p} \mathcal{O}_{X}$ is at least two.)

\section{Proof}

In the proof of Theorem 3.1, we showed $\mathrm{H}_{p}^{i}\left(f_{*} \mathcal{O}_{Y}(-E)\right) \cong \mathrm{H}_{p}^{i}\left(\mathcal{O}_{X}\right)$ for $i>1$. So for any integer $3 \leq t \leq n$, we have

$$
\begin{aligned}
\operatorname{depth}_{p} \mathcal{O}_{X} \geq t & \Leftrightarrow \quad \mathrm{H}_{p}^{i}\left(X, \mathcal{O}_{X}\right)=0, \quad \forall i<t \\
& \Leftrightarrow \quad \mathrm{H}_{p}^{i}\left(X, f_{*} \mathcal{O}_{Y}(-E)\right)=0, \quad \forall 1<i<t \\
& \Leftrightarrow \quad \mathrm{H}^{n-i}\left(E, K_{E}\right)=0, \quad \forall 1<i<t \quad \text { (Matlis duality and (3.1)) } \\
& \Leftrightarrow \quad \mathrm{H}^{i-1}\left(E, \mathcal{O}_{E}\right)=0, \quad \forall 1<i<t \quad \text { (Serre duality). }
\end{aligned}
$$




\section{REMARK 3.3}

The cohomology group $\mathrm{H}^{i}\left(E, \mathcal{O}_{E}\right)$ is independent of resolution, because $\mathrm{H}^{i}(E$, $\left.\mathcal{O}_{E}\right) \cong R^{i} f_{*} \mathcal{O}_{Y}$ by Kovács's vanishing theorem. And $R^{i} f_{*} \mathcal{O}_{Y}$ is well known to be independent of resolution.

\section{COROLLARY 3.4 ([4, PROPOSITION 4.7])}

Given a closed isolated lc center $p$ of a pair $(X, \Delta)$, then $X$ is Cohen-Macauley at $p$ if and only if $\mathrm{H}^{i}\left(E, \mathcal{O}_{E}\right)=0, \forall 0<i<n-1$.

\subsection{Normal isolated Du Bois singularity}

The notion of Du Bois singularities is a generalization of the notion of rational singularities. For a proper scheme of finite type $X$ there exists a complex $\underline{\Omega}_{X}^{\bullet}$, which is an analogue of the de Rham complex. Roughly speaking, $X$ is said to have Du Bois singularities if the natural map $\mathcal{O}_{X} \rightarrow \underline{\Omega}_{X}^{0}$ is a quasi-isomorphism. We refer the reader to [16] and the references therein for more discussions.

In this subsection we consider the case where $(X, p)$ is a normal isolated Du Bois singularity of dimension $n$ and $f: Y \rightarrow X$ is a $\log$ resolution such that $f$ is an isomorphism outside of $p$. We claim that the idea in Section 3.1 can be applied to this case. The crucial fact we need is the following.

\section{THEOREM 3.5 ([16, THEOREM 6.1])}

Take a log resolution $f: Y \rightarrow X$ as above, and let $E$ be the reduced preimage of $p$. Then $(X, p)$ is a normal Du Bois singularity if and only if the natural map

$$
R^{i} f_{*} \mathcal{O}_{Y} \rightarrow R^{i} f_{*} \mathcal{O}_{E}
$$

is an isomorphism for all $i>0$.

This theorem implies that $R^{i} f_{*} \mathcal{O}_{Y}(-E)=o, \forall i>0$. That is,

$$
f_{*} \mathcal{O}_{Y}(-E) \cong_{\text {quasi }} \mathbf{R} f_{*} \mathcal{O}_{Y}(-E) \text {. }
$$

Then exactly the same proof as in Section 3.1 yields the following theorem.

\section{THEOREM 3.6}

Given that $(X, p)$ is a normal isolated Du Bois singularity of dimension $n$, for $1<i<n, \mathrm{H}_{p}^{i}\left(X, \mathcal{O}_{X}\right)$ is dual to $\mathrm{H}^{n-i}\left(E, K_{E}\right)$ by Matlis duality. For $i=n$, $\mathrm{H}_{p}^{n}\left(X, \mathcal{O}_{X}\right)$ is dual to $f_{*} \mathcal{O}_{Y}\left(K_{Y}+E\right)$. In particular, $f_{*} \mathcal{O}_{Y}\left(K_{Y}+E\right) \cong K_{X}$.

Then the corollaries in Section 3.1 also hold.

REMARK 3.7

The last statement has been proved in [8, Claim in Theorem 2.3].

\section{Kovács's vanishing theorem}

In this section we follow Fujino's idea to give a simple proof of Kovács's vanishing theorem. First we prove a similar result for the dlt pair which was proved 
by the notion of rational pairs in [11]. One of the equivalent definitions of dlt singularities is that there is a log resolution (see Szabó's resolution in [17]) such that the discrepancy of any exceptional divisor is strictly bigger than -1 (see [13, Theorem 2.44]).

\section{THEOREM 4.1}

Let $\left(X, \Delta_{X}\right)$ be a dlt pair, and let $f: Y \rightarrow X$ be a Szabó resolution. Then we can write

$$
K_{Y}+\Delta_{Y}=f^{*}\left(K_{X}+\Delta_{X}\right)+A-B,
$$

where $A, B$ are effective exceptional divisors, $\lfloor B\rfloor=0$, and $\Delta_{Y}$ is the strict transform of $\Delta_{X}$. Then for any reduced subset $\Delta^{\prime} \subseteq \Delta_{Y}$, we have

$$
R^{i} f_{*} \mathcal{O}_{Y}\left(-\Delta^{\prime}\right)=0
$$

for every $i>0$.

Proof

Write

$$
K_{Y}-f^{*}\left(K_{X}+\Delta_{X}\right)+\Delta_{Y}=A-B
$$

Then

$$
\lceil A\rceil=K_{Y}-f^{*}\left(K_{X}+\Delta_{X}\right)+\Delta_{Y}+B+\lceil A\rceil-A,
$$

which is $f$-exceptional and effective. Consider the following diagram of complexes:

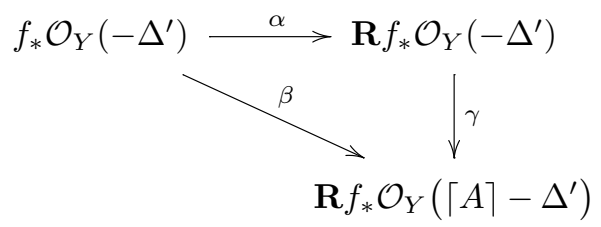

Note that

$$
\lceil A\rceil-\Delta^{\prime}=K_{Y}-f^{*}\left(K_{X}+\Delta_{X}\right)+\text { strict transform }+\delta,
$$

where $\delta$ is some effective simple normal crossing divisor such that $\lfloor\delta\rfloor=0$. So by Reid-Fukuda-type vanishing $R^{i} f_{*} \mathcal{O}_{Y}\left(\lceil A\rceil-\Delta^{\prime}\right)=0$ for $i>0$. On the other hand, since $\lceil A\rceil$ is exceptional and $\Delta^{\prime}$ is strict transform, $f_{*} \mathcal{O}_{Y}\left(\lceil A\rceil-\Delta^{\prime}\right)=f_{*} \mathcal{O}_{Y}\left(-\Delta^{\prime}\right)$ (see [10, Lemma 12]). So $\beta$ is a quasi-isomorphism.

Dualizing this diagram we have

$$
\underset{\beta^{*}}{\mathbf{R H o m}\left(f_{*} \mathcal{O}_{Y}\left(-\Delta^{\prime}\right), \omega_{X}^{\bullet}\right)<\alpha^{*}} \underset{\uparrow_{\gamma^{*}}}{\mathbf{R} \operatorname{Hom}}\left(R f_{*} \mathcal{O}_{Y}\left(\lceil A\rceil-\Delta^{\prime}\right), \omega_{X}^{\bullet}\right)
$$


Applying Grothendieck duality we get the following composition:

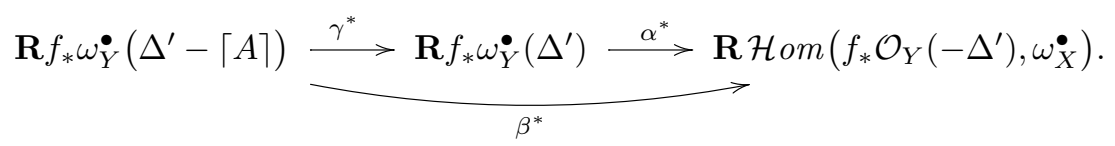

By Reid-Fukuda-type vanishing, the complex $\mathcal{R} f_{*} \omega_{Y}^{\bullet}\left(\Delta^{\prime}\right)$ has vanishing higher cohomology. Note that $\beta^{*}$ is a quasi-isomorphism, so it is in fact a composition of sheaf morphisms as follows:

$$
f_{*} \omega_{Y}\left(\Delta^{\prime}-\lceil A\rceil\right) \stackrel{\gamma^{*}}{\longrightarrow} f_{*} \omega_{Y}\left(\Delta^{\prime}\right) \stackrel{\alpha^{*}}{\longrightarrow} \operatorname{Hom}\left(f_{*} \mathcal{O}_{Y}\left(-\Delta^{\prime}\right), \omega_{X}\right) .
$$

Since $\lceil A\rceil$ is effective, $\gamma^{*}$ is injective. Because $f_{*} \omega_{Y}\left(\Delta^{\prime}\right)$ is a rank-one sheaf and the composition $\alpha^{*} \circ \gamma^{*}$ is an isomorphism, $\gamma^{*}$ is in fact an isomorphism. This implies that $\alpha^{*}$ is a quasi-isomorphism, so $\alpha: f_{*} \mathcal{O}_{Y}\left(-\Delta^{\prime}\right) \rightarrow \mathbf{R} f_{*} \mathcal{O}_{Y}\left(-\Delta^{\prime}\right)$ is also a quasi-isomorphism. That is, $R^{i} f_{*} \mathcal{O}_{Y}\left(-\Delta^{\prime}\right)=0, \forall i>0$.

With Theorem 4.1, we can prove Kovács's vanishing theorem following Fujino's [5] idea. Consider the following maps:

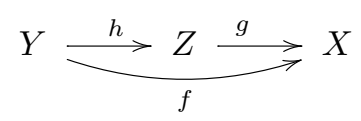

where $g:\left(Z, \Delta_{Z}\right) \rightarrow(X, \Delta)$ is a dlt modification such that $K_{Z}+\Delta_{Z}=g^{*}\left(K_{X}+\right.$ $\Delta)$. And $h: Y \rightarrow Z$ is a Szabó resolution such that $K_{Y}=h^{*}\left(K_{Z}+\Delta_{Z}\right)+A-$ $B-\Delta_{Y}$, where $\Delta_{Y}=h_{*}^{-1} \Delta_{Z}$.

We claim that $R^{i} f_{*} \mathcal{O}_{Y}\left(-\left\lfloor\Delta_{Y}\right\rfloor\right)=0, \forall i>0$. By Theorem 4.1, $R^{i} h_{*} \mathcal{O}_{Y}\left(-\left\lfloor\Delta_{Y}\right\rfloor\right)=0, \forall i>0$. Also note that $h_{*} \mathcal{O}_{Y}\left(\lceil A\rceil-\left\lfloor\Delta_{Y}\right\rfloor\right)=$ $h_{*} \mathcal{O}_{Y}\left(-\left\lfloor\Delta_{Y}\right\rfloor\right)=\mathcal{O}_{Z}\left(-\left\lfloor\Delta_{Z}\right\rfloor\right)$ (see [10, Lemma 12]). So by the Leray spectral sequence, $R^{i} f_{*} \mathcal{O}_{Y}\left(-\left\lfloor\Delta_{Y}\right\rfloor\right)=R^{i} g_{*} \mathcal{O}_{Z}\left(-\left\lfloor\Delta_{Z}\right\rfloor\right)$. The latter is zero for $i>0$ by $[9$, Theorem 1.2.5, Remark 1.2.6].

Note that $f: Y \rightarrow X$ is not a log resolution. To fix the problem, we can blow up centers with simple normal crossing with $\operatorname{Supp}\left(\Delta_{Y}+A+B\right)$. Say the blowup is $\pi: W \rightarrow Y$. There are two cases that can happen. If we blow up the klt locus, it is a Szabó resolution and then the divisor with -1 discrepancy is $\Delta_{W}=\pi_{*}^{-1}\left(\Delta_{Y}\right)$. Then $R^{i} \pi_{*} \mathcal{O}_{W}\left(-\Delta_{W}\right)=0$ by Theorem 4.1. If we blow up centers inside the non-klt locus, then the divisor with -1 discrepancy may be $\Delta_{W}=\pi_{*}^{-1}\left(\Delta_{Y}\right)+F$, where $F$ is the exceptional divisor produced by the blowup. Then $R^{i} \pi_{*} \mathcal{O}_{W}\left(-\Delta_{W}\right)=0$ by direct calculation. In any case we have shown that the higher direct image is not changed by these two kinds of blowing up. So we can conclude Kovács's vanishing theorem.

Acknowledgments. I would like to thank Professor Osamu Fujino for discussions and answering many questions by email. Moreover, this project is inspired by his paper [4]. I would also like to thank Professors Lawrence Ein, Sándor Kovács, and Mihnea Popa for many useful discussions. I am grateful to the referees for their careful reading and many useful comments. 


\section{References}

[1] V. Alexeev, Limits of stable pairs, Pure Appl. Math. Q. 4 (2008), 1-15. MR 2435844. DOI 10.4310/PAMQ.2008.v4.n3.a7.

[2] V. Alexeev and C. D. Hacon, Non-rational centers of log canonical singularities, J. Algebra 369 (2012), 1-15. MR 2959783. DOI 10.1016/j.jalgebra.2012.06.015.

[3] W. Bruns and J. Herzog, Cohen-Macaulay Rings, Cambridge Stud. Adv. Math. 39, Cambridge Univ. Press, Cambridge, 1993. MR 1251956.

[4] O. Fujino, On isolated log canonical singularities with index one, J. Math. Sci. Univ. Tokyo 18 (2011), 299-323. MR 2906532.

[5] _ A remark on Kovács's vanishing theorem, Kyoto J. Math. 52 (2012), 829-832. MR 2998913.

[6] - Introduction to the log minimal model program for log canonical pairs, preprint, arXiv:0907.1506v1 [math.AG].

[7] R. Hartshorne, Residues and Duality, with an appendix by P. Deligne, Lecture Notes in Math. 20, Springer, Berlin, 1966. MR 0222093.

[8] S. Ishii, On isolated Gorenstein singularities, Math. Ann. 270 (1985), 541-554. MR 0776171. DOI 10.1007/BF01455303.

[9] Y. Kawamata, K. Matsuda, and K. Matsuki, "Introduction to the minimal model program" in Algebraic Geometry (Sendai, 1985), Adv. Stud. Pure Math. 10, 283-360. MR 0946243.

[10] J. Kollár, A local version of the Kawamata-Viehweg vanishing theorem, Pure Appl. Math. Q. 7 (2011), 1477-1494. MR 2918170.

DOI 10.4310/PAMQ.2011.v7.n4.a18.

[11] Singularities of the Minimal Model Program, with the collaboration of Sándor J. Kovács, Cambridge Tracts in Math. 200, Cambridge Univ. Press, Cambridge, 2013. MR 3057950.

[12] J. Kollár and S. Kovács, Log canonical singularities are Du Bois, J. Amer. Math. Soc. 23 (2010), 791-813. MR 2629988.

DOI 10.1090/S0894-0347-10-00663-6.

[13] J. Kollár and S. Mori, Birational Geometry of Algebraic Varieties, with the collaboration of C. H. Clemens and A. Corti, Cambridge Tracts in Math. 134, Cambridge Univ. Press, Cambridge, 1998. MR 1658959.

DOI 10.1017/CBO9780511662560.

[14] S. Kovács, Du Bois pairs and vanishing theorems, Kyoto J. Math. 51 (2011), 47-69. MR 2784747. DOI 10.1215/0023608X-2010-020.

[15] _ Irrational centers, Pure Appl. Math. Q. 7 (2011), 1495-1515.

MR 2918171. DOI 10.4310/PAMQ.2011.v7.n4.a19.

[16] S. J. Kovács and K. Schwede, "Hodge theory meets the minimal model program: A survey of log canonical and Du Bois singularities" in Topology of Stratified Spaces, Math. Sci. Res. Inst. Publ. 58, Cambridge Univ. Press, Cambridge, 2011, 51-94. MR 2796408. 
[17] E. Szabó, Divisorial log terminal singularities, J. Math. Sci. Univ. Tokyo 1 (1995), 631-639. MR 1322695.

Department of Mathematics, Statistics, and Computer Science, University of Illinois at Chicago, Chicago, Illinois 60607, USA; cchou20@uic.edu 